\section{Modified virtual reality technology for treatment of amblyopia}

${ }^{1}$ Virtual Reality Applications Research Team (VIRART), University of Nottingham, School of 4M, University Park, Nottingham, UK

${ }^{2}$ Directorate of Ophthalmology, 'A' Floor, Eye, Ear, Nose and Throat Centre, Queen's Medical Centre, Derby Road, Nottingham, UK

Correspondence: RM Eastgate, VIRART, University of Nottingham, School of 4M, University Park, Nottingham NG7 2RD, UK Tel: + 440115951 4042; Fax: + 4401158466771 E-mail: richard.eastgate@ nottingham.ac.uk

Received: 15 November 2004

Accepted: 22 February 2005 Published online: 15 April 2005

\begin{abstract}
Purpose The conventional patching/ occlusion treatment for amblyopia sometimes gives disappointing results for a number of reasons: it is unpopular, prolonged, frequently resulting in poor or noncompliance, and also disrupts fusion. The aim of this research was to develop a novel virtual-reality (VR)-based display system that facilitates the treatment of amblyopia with both eyes stimulated simultaneously.

Methods We have adopted a multidisciplinary approach, combining VR expertise with a team of ophthalmologists and orthoptists to develop the Interactive Binocular Treatment (I-BiT ${ }^{\mathrm{TM}}$ ) system. This system incorporates adapted VR technology and specially written software providing interactive 2D and 3D games and videos to the patient via a stereo (binocular) display, and a control screen for the clinician.

Results We developed a prototype research system designed for treatment of amblyopia in children.

Conclusions The result is a novel way to treat amblyopia, which allows binocular treatment. It is interactive, and as it is partially software based, can be adapted to suit the age/ability, and needs of the patient. This means that the treatment can be made captivating and enjoyable. Further research is on-going to determine the efficacy of this new modality in the treatment of amblyopia.

Eye (2006) 20, 370-374. doi:10.1038/sj.eye.6701882; published online 15 April 2005
\end{abstract}

Keywords: amblyopia; virtual reality (VR); children

\section{Introduction}

Conventional amblyopia therapy typically consists of correction of any significant
RM Eastgate ${ }^{1}$, GD Griffiths' ${ }^{1}$ PE Waddingham ${ }^{1,2}$, AD Moody', TKH Butler2, SV Cobb', IF Comaish², SM Haworth², RM Gregson², IM Ash ${ }^{2}$ and SM Brown ${ }^{2}$ refractive error and occlusion of the nonamblyopic eye to encourage the use of and thereby stimulate the amblyopic eye. This usually entails the attachment of a sticky patch to the child's face, beneath their glasses, if worn, on a full- or part-time basis. The disadvantages of occlusion therapy for amblyopia include the significant time commitment required, both from the child for wearing the patch and parental/teacher monitoring. Furthermore, it is well documented that noncompliance results in treatment failure. ${ }^{1}$ Occlusion of the nonamblyopic eye can disrupt schooling and interfere with social activities. Poor levels of vision in the amblyopic eye can be extremely debilitating and prevent participation in enjoyable activities. Parents often find patching difficult to implement, children dislike the treatment as the patches are uncomfortable to remove, result in poorer vision while the good eye is patched, and the child often feels stigmatised. In addition occlusion therapy can be prolonged, sometimes lasting more than a year, ${ }^{2}$ and patching cannot improve binocular cooperation. ${ }^{3}$

Our aim was to develop an amblyopia treatment which allows both eyes to be used at the same time, thus improving visual acuity in an enjoyable format for children. Our group, a unique collaboration between the Directorate of Ophthalmology, Queen's Medical Centre, Nottingham and the Virtual Reality Applications Research Team (VIRART) based at the University of Nottingham has produced a prototype Interactive Binocular Treatment System (I-BiT $\left.{ }^{\mathrm{TM}}\right)$. This offers a potentially new treatment method for amblyopia by encouraging the two eyes to work together to assimilate two separate images into a coherent image. Moreover, the use of computer games and video clips seems inherently more attractive to young children than patching and so may potentially improve treatment compliance. The 
aim of this paper is to describe the design of the first research prototype of this new treatment system.

\section{Materials and methods}

\section{I-BiT system development}

Virtual reality (VR) systems typically offer a stereo image of a $3 \mathrm{D}$ virtual environment by presenting an image separately to each eye. ${ }^{4}$ Our research has investigated the application of VR technology in a completely novel way to support assessment and treatment of amblyopia. The basis of this new approach is that we can present two totally separate (but visually related) images, one to each eye independently. A range of activities (videos and computer games) has been developed to allow the visual scene to be viewed simultaneously by both eyes with presentation of central visual content preferentially to the amblyopic eye.

Some stimuli are movie clips in which the nonamblyopic eye receives peripheral stimulus in the form of the surround of the 'virtual TV' screen and surrounding visual scene. However, only the amblyopic eye can see the moving image on the 'virtual TV' screen. Other stimuli are interactive games; some elements are common to both eyes, but key elements are visible only to the amblyopic eye, so that the game is only playable if both eyes are used together (at the same time). The programs have been written specifically to motivate and hold the interest of young children. A key element of the I-BiT system is that the images presented to each eye can be moved horizontally, vertically and torsionally to correct for the angle of strabismus.

\section{The prototype research system}

\section{Computer system}

The computer system (Figure 1) uses a standard multimedia personal computer (PC) with an extra graphics card installed to connect the stereo display. The clinician uses the keyboard, mouse and main monitor to control the content of the environment and the way it is displayed to the patient and to ensure correct application of the system. The patient interacts with the environment using either a standard PC joystick or gamepad. The system has a soundcard and speakers.

\section{Treatment delivery system}

In order to provide a different image to each eye the treatment requires a true $3 \mathrm{D} /$ stereo display device of the type used in sophisticated VR systems. There are a number of suitable devices available; we investigated the use of the V8 head-mounted display made by Virtual Research but we found this to be too heavy for extended

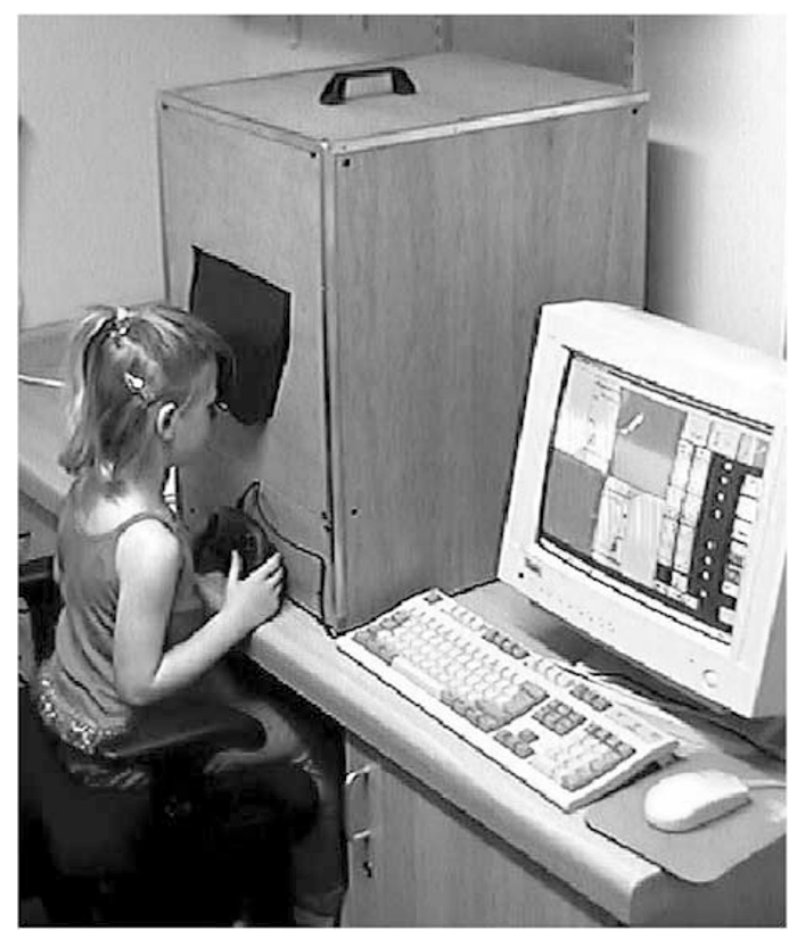

Figure 1 The computer system.

use by our expected user population. Our research system makes use of a device called a Cyberscope. This is a plastic hood that fits onto the front of a $15^{\prime \prime} \mathrm{PC}$ monitor allowing it to be used as a stereo display. As it has a stereo capability it can be used for our purposes in which the requirement is for two visually related but independent views of a visual scene to be presented to each eye separately and simultaneously. A Cyberscope mounted on a monitor standing on a desktop requires the user to look down in order to correctly view the display. Since this would not be satisfactory for our user population, we built a housing for it so that, when mounted on a standard office desk, it could be used comfortably by children between the ages of 3 and 8 years sat on an appropriate height adjustable chair (Figure 1). To reduce the size and weight of the housing, an LCD monitor was used.

\section{Software}

Figure 2 shows the control screen that gives access to and control over the various facilities provided by the software. Selection of all icons is via a mouse. This menu screen allows control by the clinician over the content of the patient display and compensation for the angle of any squint the patient may have. The right-hand side of the screen (Figure 2) shows the control buttons that the clinician can use to modify visual display parameters for each eye. The patient display can be set to show a Snellen 


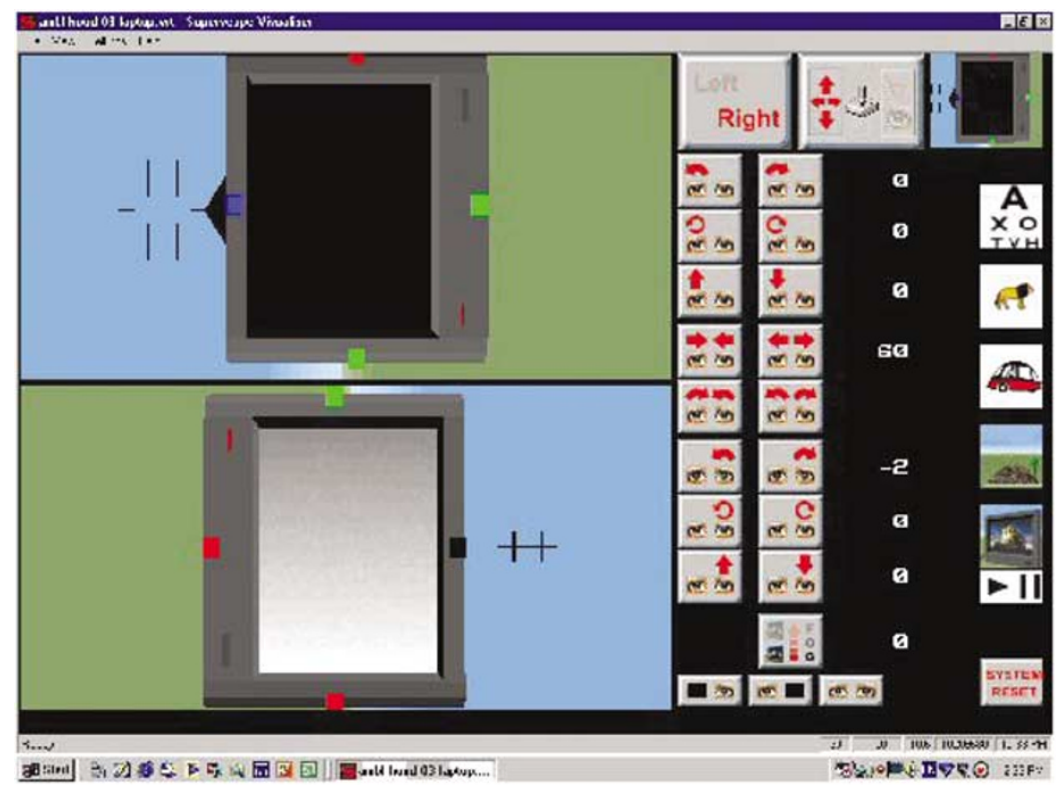

Figure 2 The practitioner's control screen.

chart, pairs of slides (features on face, hands on clock face) for establishing the patient's angle of squint and fusion range, or a viewpoint from which it is possible to watch a TV on which MPEG movie clips can be shown. The left-hand side of the screen (Figure 2) shows the clinician what is being presented to each eye via the patient's display. In the example shown in Figure 2 the right amblyopic eye is being treated. In this case, the video clip will be played on the lower of the two TV monitors (bottom left of Figure 2) and the blank TV monitor (top left of Figure 2) is presented to the nonamblyopic eye. Thus the patient views one TV monitor with the stimulus of interest being displayed to the amblyopic eye (the clinician selects the appropriate eye to be treated) and the blank TV monitor to the nonamblyopic eye. This system promotes the use of the amblyopic eye by providing central preferential interesting stimulation to this eye. The other eye receives the same view of peripheral information but no central stimulation. This system allows for both eyes to be stimulated simultaneously. This does not imply that the patient requires binocularity to be treated but that there is no occlusion of the nonamblyopic eye. The coloured squares around the screen can be used by the practitioner as a 'control' or 'tell-tale' that the patient is using both eyes, by periodically asking the patient to say what colour the squares are. If the square at the top of the screen remains blue throughout the treatment then the amblyopic and nonamblyopic eye are both being used simultaneously. If the child reports that the square at the top of the screen is black or grey, this would indicate that the fixing/nonamblyopic eye is being suppressed.
Many of the facilities provided by the system require the patient to be looking at and concentrating on the display(s) for an extended period of time (about 25 mins). However, a significant proportion of the expected user population will be aged below 10 years, and will therefore require motivation to keep them viewing the display(s). Currently, we are providing this motivation in the form of TV video programmes and interactive computer games.

\section{Video clips}

These are accessible via a menu system and watched on a TV within the environment. The practitioner can decide which of the patient's eyes will view the video, and which will just see a blank TV screen.

\section{Games}

In order to try and sustain children's interest in the experiments, it was decided to incorporate a number of interactive games into the virtual environments. The first was a version of the 1980 classic, Pac-Man, the second was a driving game.

\section{Pac-man}

Pac-man consists of a central character that must navigate around a maze avoiding the 'ghosts', while attempting to collect all the yellow 'dots'.

The very nature of the game lends itself instantly to the project and splitting it up so that a stereo version could be produced was not a complex procedure. Essentially, there are two distinct groups of components in the game; objects that move and objects that do not. We redesigned 
the game such that the amblyopic eye sees the pac-man character and ghosts, while the good eye sees the maze and the yellow dots. In order to ensure that fusion is achieved, some elements of the maze are also presented to the amblyopic eye.

\section{Racing game}

Unlike pac-man, which has an exocentric viewpoint, the racing game has an egocentric viewpoint, that is the patient viewpoint is inside, rather than outside, the user controlled object. The racing game was designed so that the two viewpoints were completely different but complementary, with each eye seeing images that constructed the full, final, image. Figure 3 shows the racing game image as presented to each eye and the composite image when both eyes are being used. In order to get the child to focus on the game, each eye is presented with half a target and alternating white lines in the centre of the road. This means that one eye sees a white line, the other eye sees the next white line, etc. In order to play the game, the child is told to focus on the target and to try and drive the car while collecting the icons within the game. There are an equal number of icons for the right eye and the left eye, this allows the clinician to confirm that the amblyopic eye is being used.

\section{Results}

The research prototype (Figure 1) was designed to allow us to conduct studies to assess the effectiveness of using $\mathrm{I}^{-B i T^{\mathrm{TM}}}$ in the treatment of amblyopia. The results of pilot trials with this system in the treatment of amblyopia in our target patient group are presented separately. ${ }^{5}$

The tolerability study showed a wide acceptability of both the equipment set up and the software content for the expected user population. However, more work is needed to develop a suite of games and activities suitable for varied users.

\section{Discussion}

The I-BiT ${ }^{\mathrm{TM}}$ system represents the first prototype VRbased computer system for the treatment of amblyopia, using dynamic stimuli with preferential stimulation of the amblyopic eye without the use of occlusion. This system was developed by a multidisciplinary team workforce whose varied and unrelated skills have taken this project in a unique direction. It is exciting to be involved in new development which avoids occlusion and has the potential to improve vision in amblyopic eyes. Our early case studies suggest promising results in children for whom previous amblyopia treatments had been unsuccessful or rejected. Details of these case study results are given in a related paper. ${ }^{5}$ However, the authors are aware that the CAM stimulator was heralded as a novel and efficacious treatment modality based on initial case studies. ${ }^{6,7}$ When a randomised controlled trial incorporated controls and a placebo treatment, visual acuity still increased. ${ }^{8}$ Their conclusions were that rotating gratings were an insignificant variable in the treatment of amblyopia. In fact visual acuity increased because occlusion was worn by the child concurrently when undertaking drawing activities on the CAM

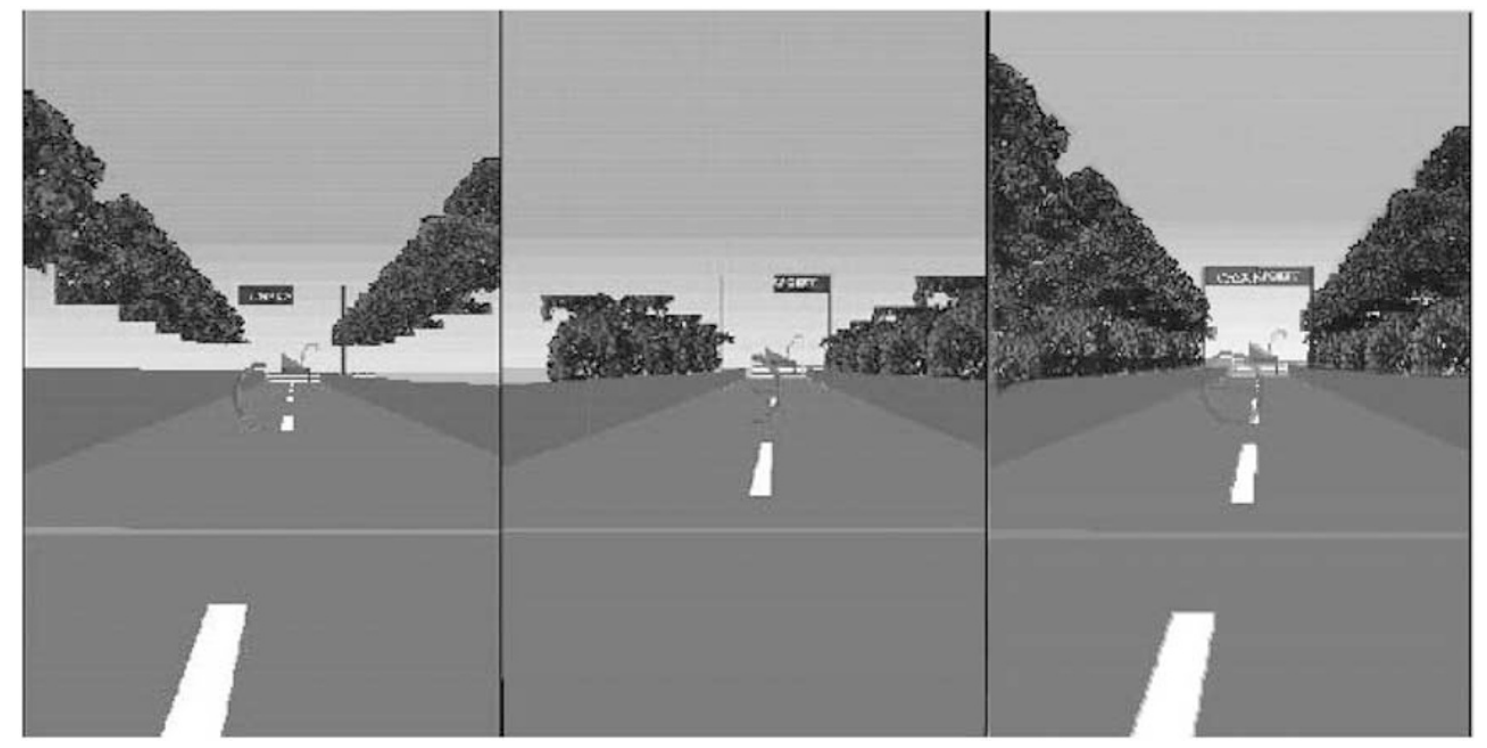

Figure 3 The racing car game. 
stimulator. However, others felt ${ }^{9}$ that the high contrast discs were the reason that the CAM treatment was unsuccessful along with the tasks the child was asked to do. It is suggested ${ }^{9}$ that low-contrast targets with high contrast flankers in conjunction with foveal targets are more efficacious and this combination is recommended for improving vision in adult amblyopia by perceptual learning. The debate regarding the neuro-physiological basis of the CAM stimulator continue but the authors take on board the fact that randomised control trials, which include a placebo treatment are necessary for future trials of the I-BiT ${ }^{\mathrm{TM}}$ system. As a result of the unknown mechanism of how our system works at a neuro-physiological level what constitutes a placebo treatment and a control is still under discussion. We would welcome contributions to this discussion. Further research is on-going to determine the tolerability and the efficacy of this new modality in the treatment of amblyopia.

\section{Acknowledgements}

After approaching a number of children's TV and film companies, Fox Media agreed to provide us with a number of suitable videos to use with the system. Research funded by William and Mabel Morris Charitable Trust and Sandra Charitable Trust, University of Nottingham and Queen's Medical Centre, NHS Trust. Based on a poster presented at the Royal College of Ophthalmologists Annual Congress, Birmingham, May 2003.

Patent: Int. Pat. App. WO 03/092482.
All of the authors are listed as inventors in the patent and therefore have a commercial interest in the product, in conjunction with the University of Nottingham and Queens Medical Centre.

Technology licensed to Carlton Optical Research Ltd.

\section{References}

1 Newsham D. Parental non-concordance with occlusion therapy. Br J Ophthalmol 2000; 84(9): 957-962.

2 Cleary M. Efficacy of occlusion for strabismic amblyopia: can optimal duration be identified? Br J Ophthalmol 2000; 84: 572-578.

3 Gregson R. Why are we so bad at treating amblyopia? Eye 2002; 16(4): 401-402.

4 Eastgate R. The structured development of virtual environments: enhancing functionality and interactivity. Doctor of Philosophy, University of Nottingham, Nottingham, 2001.

5 Waddingham PE, Butler TKH, Cobb SV, Moody AD, Comaish IF, Haworth SM et al. Preliminary results from the use of the novel Interactive Binocular Treatment (I-BiT ${ }^{\mathrm{TM}}$ ) system, in the treatment of strabismic and anisometropic amblyopia. Eye 2005, April 15 [E-pub ahead of print].

6 Campbell FW, Hess RF, Watson PG, Banks R. Preliminary results of a physiologically based treatment of amblyopia. Br J Ophthalmol 1978; 62: 748-755.

7 Willshaw HE, Malmheden A, Clarke J, Williams A, Dean L. Experience with the CAM vision stimulator: preliminary report. Br J Ophthalmol 1980; 64(5): 339-341.

8 Schor C, Gibson J, Hsu M, Mah M. The use of rotating gratings for the treatment of amblyopia: a clinical trial. Am J Optom Physiol Opt 1981; 58(11): 930-938.

9 Polat U, Ma-Naim T, Belkin M, Sagi D. Improving vision in adult amblyopia by perceptual learning. PNAS 2004; 101(17): 6692-6697. 